\title{
ASSOCIATION BETWEEN INFLAMMATORY MARKERS AND CLINICAL AND METABOLIC RISK FACTORS FOR CARDIOVASCULAR DISEASES
}

\author{
Inga Stuḳēna*, Andrejs Kalvelis*, Guntis Bahs*, Uldis Teibe*, Pēteris Tretjakovs**, \\ and Aivars Lejnieks* \\ * Rīga Stradinš̌ University, Dzirciema iela 16, Rīgā, LV-1007, LATVIA, \\ e-mail: inga.stukena@gmail.com \\ ** Institute of Experimental and Clinical Medicine, University of Latvia, O. Vācieša iela 4, Rīgā, LV-1004, LATVIA
}

Communicated by Arnis Vīksna

The inflammatory reaction plays an important role in the development of atherosclerosis. The clinical significance of the main inflammatory markers - C-reactive protein (CRP), interleukin 6 (IL6), tumour necrosis factor alpha (TNF- $\alpha$ ), plasminogen activator inhibitor 1 (PAl1), etc. - has not been fully established. CRP, IL6, TNF- $\alpha$, and PAl1 were assessed in 100 patients in terms of certain clinical indicators (sex, obesity indicators, blood pressure, and heart rate), total cholesterol (TC), low-density lipoprotein-cholesterol (LDL-C), high-density lipoprotein-cholesterol (HDL-C), triglycerides (TG), glucose, insulin, homeostasis model assessment of insulin resistance (HOMA-IR), adiponectin, and leptin levels. CRP and PAl1 levels were elevated in subjects with increased body mass index (BMI) and waist circumference. CRP correlated positively with indicators of carbohydrate metabolism and negatively with TC, HDL-C, and adiponectin. PAl1 correlated positively with insulin levels, HOMA-IR, leptin, and TG, but negatively with HDL-C. IL6 correlated negatively with TC, but TNF- $\alpha$ correlated negatively with HDL-C. Both IL6 and TNF- $\alpha$ correlated positively with leptin levels. TNF- $\alpha$ correlated with TG levels and the indicators of carbohydrate metabolism only in women. CRP and PAl1 are the most sensitive inflammatory markers; their levels were higher in adipose subjects.

Key words: inflammation, lipids, CRP, IL6, TNF- $\alpha$.

\section{INTRODUCTION}

The role of inflammation in the development of atherosclerosis can be considered proven (Ross, 1999; Fuster and Sanz, 2008; Virani et al., 2008). The various impacts of the traditional risk factors (RF) for cardiovascular diseases (CVD) - smoking, arterial hypertension (AH), dyslipidemia, obesity, etc. - on blood vessels are determined by the local inflammatory reactions in the vessels and the systemic inflammatory reaction (Fuster and Sanz, 2008; Ikonomidis at al., 2008).

Numerous body cells and tissues, especially monocytes and fatty tissues, express different mediators of inflammation in response to various irritants and changes in the body (including CVD RF) that stimulate both the local inflammatory reactions in blood vessels and the systemic inflammatory response (El-Mennyar, 2008; Virani et al., 2008). Various inflammatory factors have been studied in relation to atherosclerosis and chronic inflammatory reactions, including C-reactive protein (CRP), the interleukins (IL6, IL8, IL18, etc.), fibrinogen, soluble intracellular and vascular cell adhesion molecules, tumour necrosis factor alpha
(TNF- $\alpha$ ), macrophage and monocyte chemoattractant protein, serum amyloid A, and leptin. Many inflammatory markers are recognised as independent CVD RF (El-Mennyar, 2008; Miles et al., 2008). The assessment of inflammatory markers yields valuable information on the development of atherosclerosis. Recently, the inflammatory reactions linked to obesity have been intensively studied, especially in relation to metabolic syndrome (Caglayan et al., 2005; Calabro and Yeh, 2008; Calabro et al., 2008). Visceral obesity and metabolic syndrome are linked not only by adverse changes in lipid and glucose metabolism, but also by a pronounced inflammatory reaction. Fatty tissue is an active organ that produces cytokines, chemokines, and hormone-like substances (Caglayan et al., 2005; Gimeno and Klaman, 2005; Calabro and Yeh, 2008; Calabro et al., 2008). The activity of inflammatory markers is elevated in obese subjects, and also facilitates the development of obesity-linked metabolic and cardiovascular complications (Gimeno and Klaman, 2005; Calabro et al., 2008).

The most significant and extensively researched inflammatory markers are C-reactive protein (CRP), several inter- 
leukins (IL6, IL18, etc.), and TNF- $\alpha$. Plasminogen activator inhibitor 1 (PAI1) is also associated with the inflammatory response. These inflammatory markers reflect different inflammatory processes. However, they are also mutually interlinked to a certain extent and associated with traditional RF (Espinola-Klein et al., 2005; Gimeno and Klaman, 2005; Kahn et al., 2005; Ikonomidis et al., 2008). CRP is the most extensively studied inflammatory marker. It not only reflects the total inflammatory reaction in the body very well, but also influences the impairment of the endothelium itself (Kahn et al., 2005; Nilson 2005; Ikonomidis et al., 2008). The expression of CRP is stimulated by other inflammatory markers, such as TNF- $\alpha$ and IL6, which also have an adverse impact on endothelial function. TNF- $\alpha$ facilitates insulin resistance (Berg and Scherer, 2005; Caglayan et al., 2005; Matsuzawa, 2006) and correlates with glucose and insulin levels (Calabro and Yeh, 2008). Levels of IL6 also correlate with insulin resistance (Gimeno and Klaman, 2005; Calabro and Yeh, 2008). TNF- $\alpha$ is closely linked to dyslipidemia, because by reducing the activity of lipoprotein lipase, it stimulates the elevation of triglyceride (TG) levels and reduces high-density lipoprotein-cholesterol (HDL-C) (Rosenson, 2005).

PAI1, a regulator of the endogenous fibrinolytic system, which is predominantly expressed in the vessel walls, plays a significant role in the development of thrombotic complications, remodelling of artery walls, and CVD (Gimeno and Klaman, 2005; Taeve et al., 2005). The expression of PAI1 in the endothelium is stimulated by the inflammatory response and elevated glucose and insulin levels in the blood, and correlates with levels of IL6 and TNF- $\alpha$ (Rosenson, 2005; Taeve et al., 2005). Previous studies have reported contradictory findings regarding the role of IL6 and TNF- $\alpha$ in the development of CVD, especially in terms of their links to traditional RF (Alberti et al., 2005; El-Menyar, 2008; Ikonomidis et al., 2008).

Because of the recognised role of inflammation in the development of atherosclerosis, research into inflammatory markers is considered very promising in this context (ElMenyar, 2008; Espinola-Klein et al., 2008; Fuster and Sanz, 2008; Ikonomidis et al., 2008). The investigation of these markers should allow us to define more accurately the course and severity of atherosclerosis, and assist us in assessing CVD risk (Alberti et al., 2005; El-Menyar, 2008; Ikonomidis et al., 2008). The assessment of inflammatory markers could be especially valuable in the diagnosis of subclinical atherosclerosis (Nilson, 2005; El-Menyar, 2008; Ikonomidis et al., 2008). Changes in inflammatory markers differ over the various stages of atherosclerosis; consequently, the simultaneous assessment of many markers is of great value. The larger the number of altered markers, the greater the risk of CVD (Ikonomidis et al., 2008). Regardless of the recognised and important role of inflammatory markers in the assessment of CVD risk, their clinical meaning requires a more precise definition (Ikonomidis et al., 2008).
The objective of this work was to assess the connection between four inflammatory markers (CRP, IL6, TNF- $\alpha$, and PAI1) and the clinical RF of CVD, indicators of lipid and carbohydrate metabolism, adiponectin, and leptin.

\section{MATERIALS AND METHODS}

One hundred patients were analysed, 48 of whom were outpatients without clinical signs of CVD and 52 of whom were treated on an inpatient basis, predominantly for $\mathrm{AH}$ (48 patients) or for coronary heart disease or type 2 diabetes mellitus (four patients). All patients gave their informed consent to the protocol, which was approved by the local Medical Ethics Committee of Latvian Institute of Cardiology of University of Latvia for Medical Research. The average patient age $( \pm \mathrm{SD})$ was $52.4 \pm 11.1$ years, and $41 \%$ were male. Plasma levels of CRP $(\mathrm{mg} / \mathrm{mL})$ and IL6 $(\mathrm{pg} / \mathrm{mL})$, TNF- $\alpha(\mathrm{pg} / \mathrm{mL})$, and PAI1 (ng/mL) levels in the blood were assessed in all patients. Blood samples $(5 \mathrm{~mL})$ for cytokine analysis were collected after a $12 \mathrm{~h}$ fast and a 30 min resting period in the supine position. They were immediately centrifuged at $4{ }^{\circ} \mathrm{C}$ for $20 \mathrm{~min}$ at $1600 \times g$ to separate the serum, and the samples were stored at $-70{ }^{\circ} \mathrm{C}$. xMAP multiplex immunobead assay technology was used to measure TNF- $\alpha$, IL6, leptin, PAI1, and adiponectin with a Luminex 200 analyser (Luminex Corp., Austin, TX). To quantify insulin resistance, we used the homeostasis model assessment of insulin resistance (HOMA-IR $=$ [fasting glucose $\times$ fasting insulin]/22.5). The HOMA-IR values have been shown to correlate well with values obtained using the "gold standard" clamp technique (Bonora et al., 2000). Fasting concentrations of lipids, insulin, homocysteine, CRP, and glucose were analysed using standard methods.

These markers were assessed separately in male and female patients, and in patients with elevated BMI $\left(\geq 30 \mathrm{~kg} / \mathrm{m}^{2}\right)$ and high waist circumference (WC; $\geq 102 \mathrm{~cm}$ for men and $\geq$ $88 \mathrm{~cm}$ for women). After the data were confirmed to have a normal distribution, statistical differences between the abovementioned groups were assessed by one-way analysis of variance using Fisher's multiple comparison test. Data were recorded as the mean $\pm \mathrm{SD}$, and two-tailed values of $P<0.05$ were considered significant. Using Pearson's correlation coefficient, we analysed the correlations between inflammatory markers and patient sex, age, adiposity indicators, systolic and diastolic blood pressures (SBP and DBP, respectively), heart rate (HR), total cholesterol (TC), low-density lipoprotein-cholesterol (LDL-C), HDL-C, TG, glucose, insulin, adiponectin, and leptin levels in blood plasma and the HOMA-IR index.

\section{RESULTS}

The values obtained for the analysed inflammatory markers are listed in Table 1 according to patient sex and obesity indicators.

No significant sex-based differences in inflammatory markers were observed. In obese patients (those with increased 
LEVELS OF INFLAMMATORY MARKERS ( \pm SD) IN THE TOTAL GROUP ACCORDING TO SEX AND OBESITY INDICATORS

\begin{tabular}{|c|c|c|c|c|c|c|c|c|c|c|}
\hline & \multirow[t]{2}{*}{ Total group } & \multicolumn{3}{|c|}{ Sex } & \multicolumn{3}{|c|}{ BMI } & \multicolumn{3}{|c|}{ Waist circumference } \\
\hline & & male & female & $P$ & $<30$ & $\geq 30$ & $P$ & normal & increased & $P$ \\
\hline $\begin{array}{l}\mathrm{CRP} \\
\mathrm{mg} / \mathrm{L}\end{array}$ & $\begin{array}{c}3.85 \\
\pm 7.42\end{array}$ & $\begin{array}{c}4.86 \\
\pm 9.72\end{array}$ & $\begin{array}{c}3.15 \\
\pm 5.20\end{array}$ & 0.259 & $\begin{array}{c}2.03 \\
\pm 2.83\end{array}$ & $\begin{array}{c}6.96 \\
\pm 11.05\end{array}$ & 0.001 & $\begin{array}{c}1.54 \\
\pm 2.05\end{array}$ & $\begin{array}{c}4.84 \\
\pm 8.60\end{array}$ & 0.041 \\
\hline $\begin{array}{l}\text { IL6 } \\
\mathrm{pg} / \mathrm{ml}\end{array}$ & $\begin{array}{c}0.58 \\
\pm 1.07\end{array}$ & $\begin{array}{c}0.62 \\
\pm 1.01\end{array}$ & $\begin{array}{c}0.55 \\
\pm 1.12\end{array}$ & 0.77 & $\begin{array}{c}0.55 \\
\pm 1.14\end{array}$ & $\begin{array}{c}0.63 \\
\pm 0.97\end{array}$ & 0.705 & $\begin{array}{c}0.53 \\
\pm 0.94\end{array}$ & $\begin{array}{c}0.60 \\
\pm 1.13\end{array}$ & 0.746 \\
\hline $\begin{array}{l}\text { TNF- } \alpha \\
\mathrm{pg} / \mathrm{ml}\end{array}$ & $\begin{array}{c}3.69 \\
\pm 2.99\end{array}$ & $\begin{array}{c}3.35 \\
\pm 2.67\end{array}$ & $\begin{array}{c}3.94 \\
\pm 3.21\end{array}$ & 0.335 & $\begin{array}{c}3.29 \\
\pm 2.45\end{array}$ & $\begin{array}{c}4.38 \\
\pm 3.69\end{array}$ & 0.081 & $\begin{array}{c}3.19 \\
\pm 2.29\end{array}$ & $\begin{array}{c}3.91 \\
\pm 3.24\end{array}$ & 0.276 \\
\hline $\begin{array}{l}\text { PAI1 } \\
\mathrm{ng} / \mathrm{ml}\end{array}$ & $\begin{array}{c}5.52 \\
\pm 2.96\end{array}$ & $\begin{array}{c}5.65 \\
\pm 3.26\end{array}$ & $\begin{array}{c}5.42 \\
\pm 2.73\end{array}$ & 0.70 & $\begin{array}{c}4.24 \\
\pm 1.65\end{array}$ & $\begin{array}{c}7.69 \\
\pm 3.41\end{array}$ & 0.001 & $\begin{array}{c}4.51 \\
\pm 2.37\end{array}$ & $\begin{array}{c}5.95 \\
\pm 3.09\end{array}$ & 0.025 \\
\hline
\end{tabular}

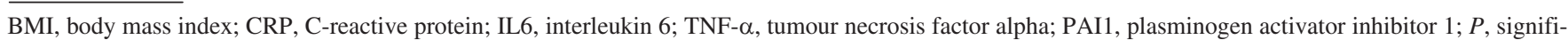
cance of diference

Table 2

CORRELATIONS BETWEEN INFLAMMATORY MAKERS AND CLINICAL INDICATORS IN THE TOTAL GROUP (T) AND SEPARATELY IN MEN AND WOMEN

\begin{tabular}{|c|c|c|c|c|c|c|c|c|c|}
\hline Marker & Group & & Age & Sex & WC & BMI & SBP & DBP & $\mathrm{HR}$ \\
\hline \multirow{3}{*}{ CRP } & $\mathrm{T}$ & $\begin{array}{c}\text { PCC } \\
P \\
\end{array}$ & $\begin{array}{l}0.007 \\
0.941 \\
\end{array}$ & $\begin{array}{c}-0.114 \\
0.259 \\
\end{array}$ & $\begin{array}{l}0.363 \\
0.000 \\
\end{array}$ & $\begin{array}{l}0.372 \\
0.000 \\
\end{array}$ & $\begin{array}{l}0.109 \\
0.282 \\
\end{array}$ & $\begin{array}{r}-0.087 \\
0.390 \\
\end{array}$ & $\begin{array}{l}0.100 \\
0.320 \\
\end{array}$ \\
\hline & Male & $\begin{array}{c}\text { PCC } \\
P \\
\end{array}$ & $\begin{array}{l}0.007 \\
0.963 \\
\end{array}$ & - & $\begin{array}{l}0.408 \\
0.008 \\
\end{array}$ & $\begin{array}{l}0.454 \\
0.003 \\
\end{array}$ & $\begin{array}{r}-0.012 \\
0.939 \\
\end{array}$ & $\begin{array}{r}-0.169 \\
0.292 \\
\end{array}$ & $\begin{array}{l}0.220 \\
0.167 \\
\end{array}$ \\
\hline & Female & $\begin{array}{c}\mathrm{PCC} \\
P \\
\end{array}$ & $\begin{array}{l}0.015 \\
0.907 \\
\end{array}$ & - & $\begin{array}{l}0.303 \\
0.020 \\
\end{array}$ & $\begin{array}{l}0.331 \\
0.010 \\
\end{array}$ & $\begin{array}{l}0.240 \\
0.067 \\
\end{array}$ & $\begin{array}{l}0.004 \\
0.975 \\
\end{array}$ & $\begin{array}{r}-0.001 \\
0.997 \\
\end{array}$ \\
\hline \multirow{3}{*}{ IL6 } & $\mathrm{T}$ & $\begin{array}{c}\mathrm{PCC} \\
P \\
\end{array}$ & $\begin{array}{c}-0.181 \\
0.072 \\
\end{array}$ & $\begin{array}{c}-0.030 \\
0.770 \\
\end{array}$ & $\begin{array}{l}0.051 \\
0.613 \\
\end{array}$ & $\begin{array}{l}0.110 \\
0.274\end{array}$ & $\begin{array}{r}-0.005 \\
0.981 \\
\end{array}$ & $\begin{array}{r}-0.019 \\
0.854 \\
\end{array}$ & $\begin{array}{l}0.088 \\
0.382 \\
\end{array}$ \\
\hline & Male & $\begin{array}{c}\text { PCC } \\
P \\
\end{array}$ & $\begin{array}{r}-0.221 \\
0.166 \\
\end{array}$ & - & $\begin{array}{r}-0.071 \\
0.660 \\
\end{array}$ & $\begin{array}{l}0.092 \\
0.566\end{array}$ & $\begin{array}{r}-0.182 \\
0.255 \\
\end{array}$ & $\begin{array}{r}-0.283 \\
0.073 \\
\end{array}$ & $\begin{array}{l}0.066 \\
0.680 \\
\end{array}$ \\
\hline & Female & $\begin{array}{c}\text { PCC } \\
P \\
\end{array}$ & $\begin{array}{c}-0.155 \\
0.242 \\
\end{array}$ & - & $\begin{array}{l}0.120 \\
0.366 \\
\end{array}$ & $\begin{array}{l}0.119 \\
0.368 \\
\end{array}$ & $\begin{array}{l}0.107 \\
0.418 \\
\end{array}$ & $\begin{array}{l}0.158 \\
0.232 \\
\end{array}$ & $\begin{array}{l}0.102 \\
0.442 \\
\end{array}$ \\
\hline \multirow{3}{*}{ TNF- $\alpha$} & $\mathrm{T}$ & $\begin{array}{c}\text { PCC } \\
P \\
\end{array}$ & $\begin{array}{l}0.054 \\
0.594 \\
\end{array}$ & $\begin{array}{l}0.097 \\
0.335 \\
\end{array}$ & $\begin{array}{l}0.124 \\
0.218 \\
\end{array}$ & $\begin{array}{l}0.180 \\
0.073 \\
\end{array}$ & $\begin{array}{l}0.025 \\
0.803 \\
\end{array}$ & $\begin{array}{c}-0.071 \\
0.481 \\
\end{array}$ & $\begin{array}{r}-0.163 \\
0.105 \\
\end{array}$ \\
\hline & Male & $\begin{array}{c}\text { PCC } \\
P \\
\end{array}$ & $\begin{array}{r}-0.229 \\
0.151 \\
\end{array}$ & - & $\begin{array}{r}-0.022 \\
0.891 \\
\end{array}$ & $\begin{array}{l}0.038 \\
0.815 \\
\end{array}$ & $\begin{array}{r}-0.177 \\
0.268 \\
\end{array}$ & $\begin{array}{r}-0.054 \\
0.739 \\
\end{array}$ & $\begin{array}{r}-0.485 \\
0.001 \\
\end{array}$ \\
\hline & Female & $\begin{array}{c}\mathrm{PCC} \\
P\end{array}$ & $\begin{array}{l}0.226 \\
0.086 \\
\end{array}$ & - & $\begin{array}{l}0.259 \\
0.047\end{array}$ & $\begin{array}{l}0.259 \\
0.048 \\
\end{array}$ & $\begin{array}{l}0.189 \\
0.152 \\
\end{array}$ & $\begin{array}{c}-0.076 \\
0.568 \\
\end{array}$ & $\begin{array}{r}-0.013 \\
0.921 \\
\end{array}$ \\
\hline \multirow{3}{*}{ PAI1 } & $\mathrm{T}$ & $\begin{array}{c}\text { PCC } \\
P \\
\end{array}$ & $\begin{array}{r}-0.071 \\
0.484 \\
\end{array}$ & $\begin{array}{r}-0.039 \\
0.700 \\
\end{array}$ & $\begin{array}{l}0.439 \\
0.000 \\
\end{array}$ & $\begin{array}{l}0.511 \\
0.000 \\
\end{array}$ & $\begin{array}{l}0.132 \\
0.191 \\
\end{array}$ & $\begin{array}{l}0.109 \\
0.279 \\
\end{array}$ & $\begin{array}{r}-0.040 \\
0.690 \\
\end{array}$ \\
\hline & Male & $\begin{array}{c}\text { PCC } \\
P \\
\end{array}$ & $\begin{array}{r}-0.292 \\
0.064 \\
\end{array}$ & - & $\begin{array}{l}0.362 \\
0.020 \\
\end{array}$ & $\begin{array}{l}0.496 \\
0.001 \\
\end{array}$ & $\begin{array}{c}0.112 \\
0.486 \\
\end{array}$ & $\begin{array}{l}0.108 \\
0.503 \\
\end{array}$ & $\begin{array}{r}-0.079 \\
0.623 \\
\end{array}$ \\
\hline & Female & $\begin{array}{c}\text { PCC } \\
P\end{array}$ & $\begin{array}{l}0.127 \\
0.336\end{array}$ & - & $\begin{array}{l}0.516 \\
0.000\end{array}$ & $\begin{array}{l}0.530 \\
0.000\end{array}$ & $\begin{array}{l}0.141 \\
0.288\end{array}$ & $\begin{array}{l}0.108 \\
0.417\end{array}$ & $\begin{array}{r}-0.011 \\
0.931\end{array}$ \\
\hline
\end{tabular}

BMI, body mass index; CRP, C-reactive protein; IL6, interleukin 6; TNF- $\alpha$, tumour necrosis factor alpha; PAI1, plasminogen activator inhibitor 1; WC, waist circumference; SBP, systolic blood pressure; DBP, diastolic blood pressure; HR, heart rate; $P$, significance of correlation

BMI and WC), elevated CRP and PAI1 were observed. An increasing trend in TNF- $\alpha(P=0.081)$ was observed in patients with high BMI.

Table 2 lists the correlations between the inflammatory markers and sex, obesity indicators, blood pressure, and HR. It follows from the data in Table 2 that CRP correlates positively with $\mathrm{WC}$ and $\mathrm{BMI}$ in the total group, and that these correlations were also significant when analysed individually in each sex. IL6 did not correlate significantly with age, obesity indicators, blood pressure, or HR in either the total group or each sex. TNF- $\alpha$ did not correlate signifi- cantly with these clinical indicators in the total group, but their analysis in each sex showed a significant negative correlation between TNF- $\alpha$ and HR only in men, and a positive correlation between TNF- $\alpha$ and the obesity indicators (WC and BMI) only in women. PAI1 correlated positively with WC and BMI in both the total group and separately in each sex.

The correlations between the studied inflammatory markers and the lipid and carbohydrate metabolism indicators, adiponectin, and leptin are listed in Table 3. PAI1 correlated significantly negatively with HDL-C and significantly posi- 
CORRELATIONS BETWEEN INFLAMMATORY MARKERS AND LIPIDS, INDICATORS OF CARBOHYDRATE METABOLISM, LEPTIN, AND ADIPONECTIN IN THE TOTAL GROUP (T) AND SEPARATELY IN MEN AND WOMEN

\begin{tabular}{|c|c|c|c|c|c|c|c|c|c|c|c|}
\hline Marker & Group & & $\mathrm{TC}$ & LDL-C & HDL-C & TG & Glucose & Insulin & HOMA IR & $\begin{array}{l}\text { Adipo- } \\
\text { nectin }\end{array}$ & Leptin \\
\hline \multirow{3}{*}{ CRP } & $\mathrm{T}$ & $\begin{array}{c}\mathrm{PCC} \\
P\end{array}$ & $\begin{array}{c}-0.223 \\
0.026 \\
\end{array}$ & $\begin{array}{c}-0.173 \\
0.086 \\
\end{array}$ & $\begin{array}{c}-0.299 \\
0.002 \\
\end{array}$ & $\begin{array}{l}0.142 \\
0.159 \\
\end{array}$ & $\begin{array}{l}0.439 \\
0.000 \\
\end{array}$ & $\begin{array}{l}0.349 \\
0.000\end{array}$ & $\begin{array}{l}0.553 \\
0.000 \\
\end{array}$ & $\begin{array}{c}-0.230 \\
0.022 \\
\end{array}$ & $\begin{array}{l}0.188 \\
0.060\end{array}$ \\
\hline & Male & $\begin{array}{c}\mathrm{PCC} \\
P\end{array}$ & $\begin{array}{c}-0.294 \\
0.062 \\
\end{array}$ & $\begin{array}{c}-0.263 \\
0.096 \\
\end{array}$ & $\begin{array}{c}-0.308 \\
0.050 \\
\end{array}$ & $\begin{array}{l}0.053 \\
0.743 \\
\end{array}$ & $\begin{array}{l}0.692 \\
0.000 \\
\end{array}$ & $\begin{array}{l}0.384 \\
0.016 \\
\end{array}$ & $\begin{array}{l}0.692 \\
0.000 \\
\end{array}$ & $\begin{array}{c}-0.238 \\
0.135 \\
\end{array}$ & $\begin{array}{l}0.250 \\
0.116 \\
\end{array}$ \\
\hline & Female & $\begin{array}{c}\text { PCC } \\
P \\
\end{array}$ & $\begin{array}{c}-0.105 \\
0.431 \\
\end{array}$ & $\begin{array}{r}-0.031 \\
0.814 \\
\end{array}$ & $\begin{array}{c}-0.303 \\
0.020 \\
\end{array}$ & $\begin{array}{l}0.254 \\
0.052 \\
\end{array}$ & $\begin{array}{l}0.164 \\
0.215 \\
\end{array}$ & $\begin{array}{l}0.273 \\
0.038 \\
\end{array}$ & $\begin{array}{l}0.279 \\
0.034 \\
\end{array}$ & $\begin{array}{c}-0.194 \\
0.141 \\
\end{array}$ & $\begin{array}{l}0.321 \\
0.013 \\
\end{array}$ \\
\hline \multirow{3}{*}{ IL6 } & $\mathrm{T}$ & $\begin{array}{c}\mathrm{PCC} \\
P \\
\end{array}$ & $\begin{array}{r}-0.214 \\
0.033 \\
\end{array}$ & $\begin{array}{r}-0.167 \\
0.097 \\
\end{array}$ & $\begin{array}{c}-0.099 \\
0.326 \\
\end{array}$ & $\begin{array}{r}-0.032 \\
0.751 \\
\end{array}$ & $\begin{array}{l}0.092 \\
0.362 \\
\end{array}$ & $\begin{array}{l}0.103 \\
0.313 \\
\end{array}$ & $\begin{array}{l}0.147 \\
0.150 \\
\end{array}$ & $\begin{array}{l}0.030 \\
0.770 \\
\end{array}$ & $\begin{array}{l}0.371 \\
0.000 \\
\end{array}$ \\
\hline & Male & $\begin{array}{c}\mathrm{PCC} \\
P \\
\end{array}$ & $\begin{array}{r}-0.198 \\
0.214 \\
\end{array}$ & $\begin{array}{r}-0.199 \\
0.213 \\
\end{array}$ & $\begin{array}{r}-0.007 \\
0.964 \\
\end{array}$ & $\begin{array}{r}-0.037 \\
0.817 \\
\end{array}$ & $\begin{array}{l}0.269 \\
0.090 \\
\end{array}$ & $\begin{array}{c}-0.004 \\
0.982 \\
\end{array}$ & $\begin{array}{l}0.203 \\
0.215 \\
\end{array}$ & $\begin{array}{l}0.112 \\
0.484 \\
\end{array}$ & $\begin{array}{l}0.218 \\
0.170 \\
\end{array}$ \\
\hline & Female & $\begin{array}{c}\text { PCC } \\
P \\
\end{array}$ & $\begin{array}{r}-0.225 \\
0.087 \\
\end{array}$ & $\begin{array}{c}-0.143 \\
0.281 \\
\end{array}$ & $\begin{array}{c}-0.139 \\
0.292 \\
\end{array}$ & $\begin{array}{c}-0.058 \\
0.660 \\
\end{array}$ & $\begin{array}{c}-0.018 \\
0.893 \\
\end{array}$ & $\begin{array}{l}0.185 \\
0.164 \\
\end{array}$ & $\begin{array}{l}0.097 \\
0.470 \\
\end{array}$ & $\begin{array}{l}0.009 \\
0.948 \\
\end{array}$ & $\begin{array}{l}0.491 \\
0.000 \\
\end{array}$ \\
\hline \multirow{3}{*}{ TNF- $\alpha$} & $\mathrm{T}$ & $\begin{array}{c}\mathrm{PCC} \\
P \\
\end{array}$ & $\begin{array}{c}-0.130 \\
0.199 \\
\end{array}$ & $\begin{array}{r}-0.017 \\
0.866 \\
\end{array}$ & $\begin{array}{c}-0.208 \\
0.038 \\
\end{array}$ & $\begin{array}{l}0.056 \\
0.582 \\
\end{array}$ & $\begin{array}{l}0.192 \\
0.058 \\
\end{array}$ & $\begin{array}{l}0.168 \\
0.100 \\
\end{array}$ & $\begin{array}{l}0.185 \\
0.069 \\
\end{array}$ & $\begin{array}{r}-0.142 \\
0.159 \\
\end{array}$ & $\begin{array}{l}0.439 \\
0.000 \\
\end{array}$ \\
\hline & Male & $\begin{array}{c}\text { PCC } \\
P \\
\end{array}$ & $\begin{array}{r}-0.171 \\
0.286 \\
\end{array}$ & $\begin{array}{r}-0.125 \\
0.436 \\
\end{array}$ & $\begin{array}{c}-0.091 \\
0.572 \\
\end{array}$ & $\begin{array}{c}-0.076 \\
0.638 \\
\end{array}$ & $\begin{array}{r}-0.102 \\
0.524 \\
\end{array}$ & $\begin{array}{l}0.021 \\
0.901 \\
\end{array}$ & $\begin{array}{c}-0.045 \\
0.786 \\
\end{array}$ & $\begin{array}{c}-0.024 \\
0.880 \\
\end{array}$ & $\begin{array}{l}0.374 \\
0.016 \\
\end{array}$ \\
\hline & Female & $\begin{array}{c}\text { PCC } \\
P \\
\end{array}$ & $\begin{array}{r}-0.127 \\
0.339 \\
\end{array}$ & $\begin{array}{l}0.034 \\
0.800 \\
\end{array}$ & $\begin{array}{r}-0.370 \\
0.004 \\
\end{array}$ & $\begin{array}{l}0.291 \\
0.026 \\
\end{array}$ & $\begin{array}{l}0.371 \\
0.004 \\
\end{array}$ & $\begin{array}{l}0.334 \\
0.010 \\
\end{array}$ & $\begin{array}{l}0.443 \\
0.000 \\
\end{array}$ & $\begin{array}{c}-0.282 \\
0.030 \\
\end{array}$ & $\begin{array}{l}0.463 \\
0.000 \\
\end{array}$ \\
\hline \multirow{3}{*}{ PAI1 } & $\mathrm{T}$ & $\begin{array}{c}\text { PCC } \\
P \\
\end{array}$ & $\begin{array}{c}-0.052 \\
0.611 \\
\end{array}$ & $\begin{array}{l}-0.064 \\
0.527 \\
\end{array}$ & $\begin{array}{c}-0.302 \\
0.002 \\
\end{array}$ & $\begin{array}{l}0.294 \\
0.003 \\
\end{array}$ & $\begin{array}{l}0.144 \\
0.154 \\
\end{array}$ & $\begin{array}{l}0.435 \\
0.000 \\
\end{array}$ & $\begin{array}{l}0.336 \\
0.001 \\
\end{array}$ & $\begin{array}{r}-0.175 \\
0.081 \\
\end{array}$ & $\begin{array}{l}0.270 \\
0.007 \\
\end{array}$ \\
\hline & Male & $\begin{array}{c}\text { PCC } \\
P \\
\end{array}$ & $\begin{array}{c}-0.045 \\
0.778 \\
\end{array}$ & $\begin{array}{c}-0.119 \\
0.458 \\
\end{array}$ & $\begin{array}{r}-0.321 \\
0.041 \\
\end{array}$ & $\begin{array}{l}0.313 \\
0.046\end{array}$ & $\begin{array}{l}0.224 \\
0.158 \\
\end{array}$ & $\begin{array}{l}0.384 \\
0.016 \\
\end{array}$ & $\begin{array}{l}0.299 \\
0.064\end{array}$ & $\begin{array}{c}-0.163 \\
0.310 \\
\end{array}$ & $\begin{array}{l}0.258 \\
0.104\end{array}$ \\
\hline & Female & $\begin{array}{c}\text { PCC } \\
P\end{array}$ & $\begin{array}{c}-0.049 \\
0.711\end{array}$ & $\begin{array}{c}-0.006 \\
0.966 \\
\end{array}$ & $\begin{array}{c}-0.329 \\
0.011\end{array}$ & $\begin{array}{l}0.291 \\
0.025\end{array}$ & $\begin{array}{l}0.075 \\
0.571\end{array}$ & $\begin{array}{l}0.487 \\
0.000\end{array}$ & $\begin{array}{l}0.375 \\
0.004\end{array}$ & $\begin{array}{c}-0.186 \\
0.158 \\
\end{array}$ & $\begin{array}{l}0.355 \\
0.006\end{array}$ \\
\hline
\end{tabular}

TC, total cholesterol; LDL-C, low-density lipoprotein-cholesterol; HDL-C, high-density lipoprotein-cholesterol; TG, triglycerides; BMI, body mass index; CRP, C-reactive protein; IL6, interleukin 6; TNF- $\alpha$, tumour necrosis factor alpha; PAI1, plasminogen activator inhibitor 1; WC, waist circumference; SBP, systolic blood pressure; DBP, diastolic blood pressure; $P$, significance of correlation

tively with TG. CRP correlated negatively with HDL-C and TC. IL6 correlated negatively only with TC, TNF- $\alpha$ correlated negatively with HDL-C IL6, TNF- $\alpha$, and PAI1 correlated positively with leptin, but CRP correlated negatively with adiponectin. PAI1 also tended to correlate with adiponectin, CRP, and leptin, but not significantly. When the analysis was performed separately for each sex, CRP correlated significantly with leptin in women. TNF- $\alpha$ also correlated with HDL-C and TG only in women.

CRP correlated positively with all the studied indicators of carbohydrate metabolism, but PAI1 correlated only with insulin levels and HOMA-IR. IL6 and TNF- $\alpha$ did not correlate with these indicators. In women, a significant mutual correlation was observed between all the indicators of carbohydrate metabolism: serum glucose, insulin, and the HOMA-IR index.

\section{DISCUSSION}

Although the results of previous studies (Khera et al., 2005; Saltevo et al., 2008) indicate that CRP levels are higher in women, especially in women with metabolic syndrome, we observed no significant sex-specific changes in or correlations between CRP, IL6, TNF- $\alpha$, and PAI1. Among the studied inflammatory markers, only CRP and PAI1 were el- evated in obese patients and correlated with BMI and WC. IL6 and TNF- $\alpha$ were not significantly altered in obese patients. Previous studies report increased CRP (Berg and Scherer, 2005) and PAI-1 (Taeve et al., 2005) in adipose patients, but also increased IL6 and TNF- $\alpha$ (Gimeno and Klaman, 2005; Rosenson, 2005; Calabro and Yeh, 2008). We observed a correlation between TNF- $\alpha$ and obesity only in women. Our data indicate that CRP and PAI1 are the most sensitive markers of CVD risk, especially in obese patients. In women, the assessment of TNF- $\alpha$ could also be significant.

Although previous studies have reported correlations between CRP (Espinola-Klein et al., 2008) IL6 (Miles et al., 2008) and even PAI1 ((Bilgili et al., 2008) and blood pressure, we observed no correlation between inflammatory markers and SBP, DBP, or HR.

According to the literature, the links between inflammatory markers and lipid levels are indirect and complicated. Only TNF- $\alpha$ directly reduces the activity of lipoprotein lipase, and stimulates an increase in TG, a reduction in HDL-C, and the development of small, dense LDL particles (Rosenson, 2005).

When assessing the correlation between inflammatory markers and lipids, we found that CRP was inversely corre- 
lated with TC and HDL-C in the whole group, whereas IL6 correlated inversely only with TC. TNF- $\alpha$ correlated negatively only with HDL-C. CRP and IL-6 tended to correlate negatively with LDL-C. The link between these two inflammatory markers and seemingly favourable antiatherogenic TC and LDL-C changes, and with a simultaneous unfavourable reduction in serum HDC-L, is evidence of the complex association between inflammation and lipids. Furthermore, in patients with diabetes mellitus and metabolic syndrome, dyslipidemia is characterised not by increased LDL-C, but by the development of small, dense LDL-C particles. PAI1 exhibited a significant negative correlation with HDL-C, but no direct correlation with TG levels. Therefore, the link between PAI1 and atherogenic dyslipidemia (increased TG and reduced HDL-C) was most pronounced. Although we observed no correlation between TNF- $\alpha$ and TG levels in the total group, we identified this correlation in women. Previous studies have also demonstrated a link between TNF- $\alpha$ and atherogenic dyslipidemia (Rosenson, 2005). The negative correlation between CRP and HDL-C supports the extensively debated anti-inflammatory activity of HDL-C (Alber et al. 2008; Florentin et al., 2008). Our observations show that only HDL-C had a frequent and pronounced inverse correlation with the inflammatory markers CRP and TNF- $\alpha$, and with PAI1. This finding confirms the proposition that HDL-C is an independent CVD RF with anti-inflammatory activity (Alber et al. 2008; Florentin et al., 2008). HDLP-C showed no correlation only with IL6, and IL6 showed the least pronounced correlations with lipids (we observed only a negative correlation with TC).

The correlation between the indicators of carbohydrate metabolism and the inflammatory markers was most stable for CRP (which correlated with glucose, insulin, and HOMA-IR), whereas PAI1 correlated only with insulin levels and HOMA-IR. Previous studies report that TNF- $\alpha$ facilitates insulin resistance (Rosenson, 2005; Pi-Sinyer, 2006) and correlates with glucose and insulin levels (Calabro and Yeh, 2008). However, we observed no correlation between TNF- $\alpha$ and any of these indicators. Although no correlation was observed between TNF- $\alpha$ and the indicators of carbohydrate metabolism in the total group, we found a correlation between TNF- $\alpha$ and all three indicators in women. We also found no link between IL6 and glucose metabolism or insulin resistance, although these are reported in the literature (Gimeno and Klaman, 2005; Calabro and Yeh, 2008).

The pronounced correlation between CRP and the indicators of glucose metabolism supports the proposition that the increased CRP levels in patients with metabolic syndrome are associated with a risk of developing type 2 diabetes mellitus (Nilson, 2005).

Of the two basic adipokines (adiponectin and leptin) examined, leptin, which is itself recognised as a factor that stimulates inflammation, had a pronounced positive correlation with the inflammatory markers. The literature also contains references to the link between TNF- $\alpha$ and leptin (Pi-Sunyer,
2006; Lago et al., 2007). Adiponectin exhibited an inverse correlation only with CRP. Although it has been suggested that TNF- $\alpha$ inhibits the synthesis of adiponectin (Matsuzawa, 2006), we observed no correlation between TNF- $\alpha$ and adiponectin.

The positive correlation between inflammatory markers (especially CRP) and the indicators of carbohydrates metabolism, as well as their probable link with the development of insulin resistance and diabetes mellitus, and the negative correlation between CRP and TNF- $\alpha$ with HDL-C provides evidence of an association between these inflammatory markers and the development of atherosclerosis.

The expression of PAI1 occurs predominantly and locally in the endothelium and platelets, and systemically in the liver and fatty tissue. This expression is influenced by many factors and plays significant roles in remodelling blood vessels and thrombotic complications (Taeve et al., 2005). Our observations show an association between PAI1 with both obesity and adverse changes in lipid and carbohydrate metabolism. Therefore, PAI1 can be considered a significant marker for the development of atherosclerosis.

In conclusion, our study shows that leptin levels correlate with all the studied inflammatory markers. PAI1 and CRP are the most sensitive integral inflammatory markers. The roles of TNF- $\alpha$ and IL6 in the assessment of CVD risk should be defined more precisely in future research.

Inflammatory markers provide valuable additional information in the assessment of CVD risk. However, because the various inflammatory markers characterise different inflammatory reactions, it is important to determine several factors simultaneously.

\section{ACKNOWLEDGEMENTS}

The work was supported by the National Research Programme in Medicine 2006-2009, project nr. 9, „Prospective follow-up programme (registry) on risk factors and clinical forms of the cardiometabolic syndrome".

\section{REFERENCES}

Alber, H.F., Wanitschek, M.M., de Waha, S., Ladurner, A., Suessenbacher, A., Dorler, J., Dichtl, W., Frick, M., Ulmer, H., Pachinger, O., Weidinger, F. (2008). High-density lipoprotein cholesterol, C-reactive protein, and prevalence and severity of coronary artery disease in 5641 consecutive patients undergoing coronary angiography. Eur. J. Clin. Invest., 38(6), 372-380.

Alberti, K.G.M., Zimmet, P., Shaw, J. (2005). The metabolic syndrome - a new world wide definition. Lancet, 366, 1059-1062.

Berg, A.H., Scherer, P.E. (2005). Adipose tissue, inflammation, and cardiovascular disease. Circ. Res., 96, 939-949.

Bilgili, S.C.A., Dogan, A., Karaca, B. (2008). Inverse relationship between adiponectin and plasminogen activator inhibitor-1 in metabolic syndrome patients. Endocr. Regul., 42(1), 63-68.

Bonora, E., Targher, G., Alberiche, M., Bonadonna, R.C., Saggiani, F., Zenere, M.B., Monauni, T., Muggeo, M. (2000). Homeostasis model assessment closely mirrors the glucose clamp technique in the assessment of 
insulin sensitivity: Studies in subjects with various degrees of glucose tolerance and insulin sensitivity. Diabetes Care, 23(1), 57-63.

Caglayan, E.C., Blaschke, F., Takata, Y., Hsueh, W.A. (2005). Metabolic syndrome - interdependence of the cardiovascular and metabolic pathways. Curr. Opin. Pharmacol., 5, 135-142.

Calabro, P., Limongelli, G., Pacileo, G., Di Salvo, G., Golino, P. (2008). The role of adiposity as a determinant of an inflammatory milieu. $J$. Cardiovasc. Med., 9(5), 450-460.

Calabro, P., Yeh, E.T.H. (2008). Intra-abdominal adiposity, inflammation, and cardiovascular risk: New insight into global cardiometabolic risk. Curr. Hypertens. Rep., 10, 32-38.

El-Menyar, A.A. (2008). Cytokines and coronary artery disease: The state of the art. Crit. Pathw. Cardiol., 7(2), 139-151.

Espinola-Klein, Ch., Rupprecht, H.J., Bickel, Ch., Lackner, K., Genth-Zotz, S., Post, F., Munzel, T., Blankenberg, S. (2008). Impact of inflammatory markers on cardiovascular mortality in patients with metabolic syndrome. Eur. J. Cardiovasc. Prev. Rehab., 15, 278-284.

Florentin, M., Liberopoulus, E.N., Wierzbicki, A.S., Mikhailidis, D.P. (2008). Multiple actions of high-density lipoprotein. Curr. Opin. Cardiol., 23, 370-378.

Fuster, V., Sanz, J. (2008). Atherothrombosis: Past, present and future. Revista Argentina de Cardiologia, 76, spec. ed., 51-54.

Gimeno, R.E., Klaman, L.D. (2005). Adipose tissue as an active endocrine organ: Recent advances. Curr. Opin. Pharmacol., 5, 122-128.

Ikonomidis, I., Stamatelopoulos, K., Lekakis, J., Vamvakou, G.D., Kremastinos, D.Th. (2008). Inflammatory and non-invasive vascular markers: The multimarker approach for risk stratification in coronary artery disease. Atherosclerosis, 199, 3-11.

Kahn, R., Buse, J., Ferrannini, E., Stern, M. (2005). The metabolic syndrome: Time for a critical cppraisal. Diabetes Care, 28(9), 2289-2304.
Khera, A., Mc Guire, D.K., Murphy, A.S., Stanek, H.S., Das, S.R., Vongpatanasin, W., Wians, F.H., Jr, Grundy, S.M., Lemos, J.A. (2005). Race and gender differences in C-reactive protein levels. J. Amer. Coll. Cardiol, 46(3), 464-469.

Lago, F., Diequez, C., Gomez-Reino, J., Gualillo, O. (2007). Adipokines as emerging mediators of immune response and inflammation. Nat. Clin. Practice Rheumatol., 3(12), 716-725.

Matsuzawa, Y. (2006). The metabolic syndrome and adipocytokines. FEBS Letters, 580, 2917-2921.

Miles, E.A., Rees, D., Banerjee, T., Cazzola, R., Lewis, S., Wood, R., Oates, R., Tallant, A., Cestaro, B., Yaqoob, P., Wahle, K.W., Calder, P.C. (2008). Age related increases in circulating inflammatory markers in men are independent of BMI, blood pressure and blood lipid concentrations. Atherosclerosis, 196, 298-305.

Nilson, J. (2005). CRP-marker or marker of cardiovascular disease? Arterioscler. Thromb. Vasc. Biol., 25, 1527-1528.

Pi-Sunyer, F.X. (2006). The relation of adipose tissue to cardiometabolic risk. Clin. Cornerstone, 8 (Suppl. 4), S14-S23.

Rosenson, R.S. (2005). New approaches in the intensive management of cardiovascular risk in the metabolic syndrome. Curr. Probl. Cardiol., 30(5), 241-280.

Ross, R. (1999). Atherosclerosis — An inflammatory disease. New Engl. J. Med., 340, 115-126.

Saltevo, J., Vanhala, M., Kautiainen, H., Kumpusalo, F., Laakso, M. (2008). Gender difference in C-reactive protein, interleukin-1 receptor antagonist and adiponectin levels in metabolic syndrome: A population-based study. Diabetes Med., 25(6), 747-750.

Taeye, B.D., Smith, L.H., Vaughan, D.E. (2005). Plasminogen activator inhibitor-1 a common denominator in obesity, diabetes and cardiovascular disease. Curr. Opin. Pharmacol., 5, 149-154.

Virani, S.S., Polsani, V.R., Nambi, V. (2008). Novel markers of inflammation in atherosclerosis. Curr. Atheroscl. Rep., 10(2), 164-170.

Received 11 July 2009

\section{IEKAISUMA MARĶIERU SAISTĪBA AR KARDIOVASKULĀRO SLIMĪBU KLĪNISKAJIEM UN METABOLAJIEM RISKA FAKTORIEM}

Iekaisumam ir svarīga loma aterosklerozes attīstībā. Tomēr galveno iekaisuma marķieru - C-reaktīvā olbaltuma (CRO), interleikīna 6 (IL6), audzēja nekrozes faktora alfa (ANF- $\alpha$ ), plazminogēna aktivatora inhibitora 1 (PAI1) un citu klīniskā nozīme nav pilnība skaidra. Simt pacientiem tika noteikti CRO, IL6, ANF- $\alpha$ un PAI1 vienlaicīgi ar klīniskiem parametriem (dzimumu, adipozitātes rādītājiem, asins spiedienu un sirds frekvenci), kopējā holesterīna (KH), zema blīvuma lipoproteīnu holesterīna (ZBLH), augsta blīvuma lipoproteīnu holesterīna (ABLH), triglicerīdu (TG), glikozes, insulīna, insulīna rezistences rādītāja HOMA-IR, adiponektīna un leptīna līmeņiem. CRO un PAI1 līmeṇi bija paaugstināti personām ar palielinātu ḳermeṇa masas indeksu (ḲMI) un vēdera apkārtmēru (VA). CRO pozitīvi korelēja ar og̣̣hidrātu metabolisma rādīiāijiem un negatīvi ar KH, ABLH un adiponektinu. PAI1 pozitīvi korelēja ar insulīna līmeni, HOMA-IR, leptīnu un TG, bet negatīvi ar ABLH. IL6 negatīvi korelēja ar KH, bet ANF- $\alpha$ negatīvi korelēja ar ABLH. Bet gan IL6, gan ANF- $\alpha$ pozitīvi korelēja ar leptīna līmeni. Savukārt TG līmeni un og̣̣hidrātu metabolisma rādītājiem, ANF- $\alpha$ korelēja tikai sievietēm. CRO un PAI1 ir visjūtīgākie iekaisuma marḳieri, to līmenis ir augstāks adipozām personām. 